\title{
Desenvolvimento, Utilização e Avaliação de uma Aplicação Móvel para Educação Médica: um Estudo de Caso em Anestesiologia
}

\author{
Ricardo Victor Soares Pereira, Centro Universitário Unichristus, rvictorbr@ yahoo.com.br \\ Marcos Kubrusly, Centro Universitário Unichristus, mmkubrusly@ gmail.com \\ Edgar Marçal, Universidade Federal do Ceará, edgar@virtual.ufc.br
}

Resumo. Atualmente, as pesquisas mostram que existe uma grande quantidade de aplicações móveis sendo utilizadas para o ensino de Medicina. Porém, a maioria dos estudos encontrados não descreve os passos para a construção desses aplicativos e, em alguns casos, são usadas tecnologias inadequadas ou insuficientes que podem prejudicar os objetivos de aprendizagem. Este estudo investigou como se deu o processo de construção, utilização e avaliação em campo de uma aplicação móvel para o ensino em Anestesiologia. Durante uma semana, foi realizado um estudo de caso em um hospital, envolvendo 20 participantes (alunos de Medicina e residentes). Os resultados indicaram que a aplicação apresentava alto nível de usabilidade e foi considerada como uma ferramenta útil para treinamento e sistematização da avaliação pré-operatória.

Palavras-chave: Mobile Learning, Educação Médica e Anestesiologia.

\section{Development, Use and Evaluation of a Mobile Application for Medical Education: a Case Study in Anesthesiology}

\begin{abstract}
Currently, research shows that there are a lot of mobile applications being used for teaching medicine. However, most existing studies do not describe the steps for developing these applications and, in some cases, inadequate or insufficient technologies are used that may undermine learning objectives. This study investigated how the process of construction, use and evaluation in the field of a mobile application for the teaching in Anesthesiology. For this purpose, during a week, a case study was carried out in a hospital involving 20 participants (medical students and residents). The results indicated that the application presented a high level of usability and was considered as a useful tool for training of the preoperative evaluation.
\end{abstract}

Keywords: Mobile Learning, Medical Education and Anesthesiology.

\section{INTRODUÇÃO}

O internato ou estágio curricular obrigatório médico consiste no período de ensino prático, no final da graduação em Medicina, no qual o estudante deve receber treinamento intensivo contínuo em instituição de saúde. Já a residência médica, consiste numa modalidade de ensino de pós-graduação, onde médicos acompanham casos reais, adquirindo experiência e conhecimentos técnico-científicos essenciais em uma determinada área. Em ambos os casos, esses aprendizes constroem conhecimentos na prática em instituições hospitalares, fora de sala de aula.

Nesse sentido, as tecnologias móveis (e.g. smartphones, tablets, redes sem fio, sensores) têm se destacado como importantes ferramentas para apoio às atividades de aprendizagem em espaços não escolares. A inserção desses recursos nos processos de ensino e aprendizagem pode proporcionar diferentes benefícios (Sung; Hwang; Chang, 2016; Marçal, Andrade; Viana, 2015), tais como: a identificação do contexto do aluno 
para entrega de conteúdos e exercícios de acordo com a situação dele naquele momento; o registro das ações e preferências dos aprendizes para recomendações futuras em atividades similares; a utilização de sensores (embutidos ou acoplados ao dispositivo móvel) para anotação e sugestão de informações relevantes; e, a comunicação entre alunos e professores através de redes sem fio para resolução de dúvidas ou compartilhamento de ideias.

Por um lado, atualmente, existe uma grande quantidade de aplicações móveis relevantes para o ensino de Medicina (Briz-Ponce et al., 2016) e parece haver uma mobilização cada vez maior para se promover o uso dos dispositivos móveis nas mais diversas áreas médicas (Fuller; Joynes, 2015). Porém, por outro lado, a maioria dos trabalhos existentes não aborda os detalhes do processo de construção desses aplicativos. Desta forma, tais estudos não servem como referência para professores de Medicina e suas equipes no desenvolvimento de novas soluções, o que pode causar repetições de erros de projeto e perda de produtividade. Além disso, o uso de tecnologias inadequadas ou insuficientes pode prejudicar os objetivos de aprendizagem traçados com o uso da aplicação móvel (Walsh, 2015).

Este artigo descreve o processo de desenvolvimento, a utilização e a avaliação de uma aplicação móvel voltada para apoiar a formação de internos e residentes na área de Anestesiologia. Pretendeu-se avaliar os aspectos envolvidos na construção de uma aplicação móvel para Educação Médica através de uma metodologia de desenvolvimento que permitisse a participação efetiva dos especialistas de domínio na equipe de projeto do sistema. Após isso, buscou-se investigar se a aplicação resultante apresentava boas taxas de usabilidade e utilidade percebidas pelos aprendizes. Como tema do aplicativo, foi escolhido o procedimento de avaliação pré-operatória, por ser um assunto importante para a área de Anestesiologia e por ainda ser realizado através de formulários impressos em muitos hospitais. Este estudo faz parte de uma pesquisa de mestrado, sendo os autores, em ordem: o mestrando, o orientador e co-orientador.

\section{EMBASAMENTO TEÓRICO}

Esta seção aborda os principais assuntos que representam a base teórica deste artigo, destacando-se os conceitos relacionados ao uso dos dispositivos móveis na educação médica, as metodologias de desenvolvimento de aplicações móveis e as abordagens para avaliação de softwares.

\subsection{Tecnologias Móveis na Educação Médica}

O paradigma Mobile Learning (ou m-Learning) surgiu a partir da utilização das tecnologias da computação móvel (e.g. smartphones, tablets, redes sem fio) como parte de um modelo de aprendizado integrado (Marçal; Andrade; Rios, 2005). Com a adição das tecnologias de sensores, as alternativas para uso das ferramentas móveis em favor do ensino e aprendizagem são ampliadas, como por exemplo, torna-se possível a recomendação de conteúdos e atividades para os alunos de acordo com a localização em que eles se encontram (Spínola; Travassos, 2012).

Ao se utilizar os recursos da computação móvel para melhorar os processos na área da saúde, emprega-se o termo Mobile Health (ou m-Health). As soluções de mHealth têm proporcionado avanços nas técnicas tradicionais de monitoramento e alerta de saúde, coleta de dados clínicos e administrativos, manutenção nos registros dos pacientes e nos sistemas de detecção, prevenção, falsificação e roubo de drogas (Silva et al., 2015). 
Os dispositivos móveis têm sido utilizados pelos médicos para diversos fins, dentre os quais se destaca o uso na educação médica, quando às vezes se emprega o termo Mobile Medical Education (Davies et al., 2012). Vários estudos vêm demonstrando que a utilização das tecnologias móveis e sem fio pode proporcionar inúmeros benefícios tanto para professores quanto para estudantes de Medicina. Segundo Briz-Ponce et al. (2016), estudantes de Medicina que utilizaram uma aplicação móvel com visualização 3D sobre estruturas e funções da mente humana tiveram um desempenho superior aos alunos que não utilizaram o aplicativo. A Figura 1 resume os termos abordados nessa seção sobre a utilização dos recursos da Computação Móvel em diferentes áreas.

\section{Computação} Móvel
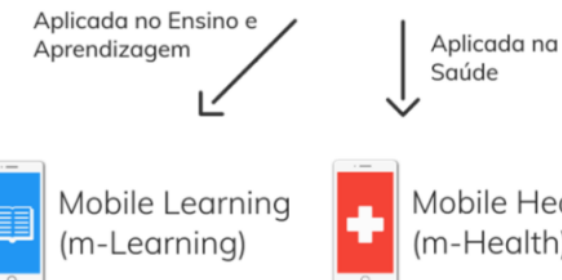

Aplicada no Ensino e

Aprendizagem de Medicino

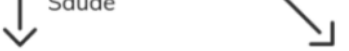

(m-Learning)

Mobile Health

(m-Health)

Mobile Medical

Education

Figura 1 - Comparação entre os termos Mobile Learning, Mobile Health e Mobile Medical Education.

\subsection{Desenvolvimento de Aplicações Móveis}

A construção de sistemas móveis apresenta desafios que precisam ser contornados, tais como: o tratamento de forma transparente da heterogeneidade dos dispositivos; a intermitência de infraestrutura de comunicação; a dinamicidade dos elementos computacionais do ambiente; e o suporte à mobilidade dos usuários (Gilman et al., 2015; Hwang e Wong, 2014). Para facilitar o desenvolvimento desses sistemas e proporcionar ganhos de produtividade e qualidade, diferentes soluções têm sido utilizadas.

Com relação às tecnologias utilizadas para desenvolvimento de aplicações móveis, Preuveneers e Novais (2012) apresentam uma análise comparativa, destacandose: padrões de projeto, middlewares, linhas de produto de software e frameworks. Os autores concluem afirmando que o desenvolvimento de sistema móveis não é uma tarefa trivial e que para implementá-los faz-se necessária a adoção de uma metodologia de reuso de forma sistemática que proporcione benefícios tanto em tempo de projeto como de execução também

Entretanto, antes da escolha da tecnologia específica para o desenvolvimento, é importante a definição do processo completo de construção do sistema, desde a identificação dos requisitos até a disponibilização para os usuários finais. Uma das abordagens mais utilizadas para o desenvolvimento de sistemas educativos é modelo ADDIE (Branch, 2009), o qual é composto por uma série de passos iterativos: Analyze, Design, Develop, Implement e Evaluate. Shuib et al. (2015) utilizaram o modelo ADDIE para guiar o desenvolvimento de uma aplicação móvel para ensino de gramática da língua inglesa.

Outra abordagem para construção de aplicações móveis é o modelo de desenvolvimento Co-Design (Millard et al., 2009), que tem como principal diferencial a participação efetiva dos especialistas de domínio na equipe de projeto do sistema. Os 
autores defendem que o formato de interação proposto pelo Co-Design facilita o levantamento dos requisitos e proporciona a criação de aplicações de $m$-Learning mais sofisticadas e mais próximas aos usuários finais. O’Connor e Andrewsb (2016) utilizaram uma adaptação do modelo Co-Design com formação de grupos focais para criar uma aplicação móvel para ensino de enfermagem mais fiel às necessidades de aprendizagem dos enfermeiros.

\subsection{Avaliação de Softwares}

Segundo Mack e Nielsen (1994), os métodos para avaliação de software podem ser agrupados em duas categorias principais: Analíticos (também conhecidos como métodos de inspeção ou heurísticas), que são aqueles nos quais a avaliação do software é baseada no julgamento de especialistas; e, Empíricos, que são aqueles que envolvem a participação dos usuários para a coleta dos dados, que posteriormente serão analisados.

Entre as técnicas utilizadas para avaliação com usuários, destaca-se a aplicação de questionários padronizados. As principais vantagens da utilização desses instrumentos para avaliação são: objetividade na coleta das informações, replicabilidade do instrumento em outros estudos e quantificação dos resultados a partir das respostas dos participantes, através de cálculos estatísticos (Sauro; Lewis, 2012).

Um dos questionários mais utilizados na avaliação de softwares é o SUS (System Usability Scale), que se caracteriza como um método de fácil aplicação para averiguação da usabilidade de sistemas (Sauro, 2011). Ele é composto por 10 questões (ou itens), cada uma com cinco opções de respostas que seguem a escala Likert de 5 pontos. Outro questionário utilizado para avaliação de softwares é o Modelo de Aceitação de Tecnologia de Davis (em inglês Davis' Technology Acceptance Model TAM), que permite a quantificação do grau de utilidade percebida pelos usuários de um determinado sistema (Wallace; Sheetz, 2014).

Além desses instrumentos, existem outros que podem ser utilizados para avaliação de softwares. Portanto, a composição das perguntas que irão compor o questionário de avaliação irá variar de acordo com os objetivos da pesquisa e quais características do software pretende-se verificar.

\section{MATERIAIS E MÉTODOS}

Esta seção descreve a metodologia utilizada neste estudo, dividindo-a nas seguintes três subseções: descrição do processo de desenvolvimento de uma aplicação móvel voltada para apoiar a formação de alunos de medicina e residentes na área de Anestesiologia; detalhamento de como se deu o uso dela pelos estudantes e especialistas; e, apresentação dos procedimentos para avaliação da usabilidade e utilidade da aplicação.

\subsection{Processo de Desenvolvimento}

A construção do aplicativo contou com a participação de diferentes profissionais: um médico anestesiologista e professor do curso de Medicina, um analista de sistema, um programador e um designer gráfico. Considerando a composição multidisciplinar das equipes em projetos de software na área médica, como nesse caso, e com o objetivo de se produzir uma aplicação mais próxima às necessidades dos usuários finais, optou-se pela utilização da metodologia Co-Design. A construção da aplicação 
seguiu as cinco fases propostas por esse modelo, com algumas adaptações necessárias. A Figura 2 ilustra o processo de desenvolvimento, descrito a seguir.

I.Escopo. Nessa fase, uma visão geral dos objetivos da aplicação foi definida, destacando-se as questões de aprendizagem sobre o procedimento da avaliação préoperatória. Outra tarefa importante nessa fase foi garantir o envolvimento de todos os stakeholders relacionados ao domínio do problema. Nesse estudo, o professor de Medicina (primeiro autor desse artigo) que participou do projeto também era Anestesiologista. Assim, pode colaborar com conhecimentos tanto sobre a educação médica quanto na área específica da aplicação.

II.Compreensão Compartilhada. Nessa fase, os stakeholders trocaram experiências e relacionaram: os possíveis atores e cenários nos quais a aplicação poderia ser utilizada; os tipos de tecnologias que eram usadas em soluções similares; e as metodologias pedagógicas que podiam servir de base para implementação da aplicação.

III.Brainstorming. Nessa fase, foi possível esboçar as primeiras interfaces da aplicação, considerando os atores, cenários, tecnologias e metodologias pedagógicas identificadas na etapa anterior. Com isso, os stakeholders contavam com artefatos mais próximos à aplicação final para colaborar com sugestões.

IV.Refinamento. À medida que a aplicação ia ganhando uma aparência final e o levantamento dos requisitos era concluído, seguiu-se para modelagem dos diagramas do Projeto (e.g, Casos de Uso, Diagrama de Classes, Diagrama de Atividades).

V.Implementação. Após a definição dos modelos, partiu-se para o desenvolvimento iterativo da aplicação com entregas incrementais. É importante destacar que as fases III, IV e V aconteceram de forma cíclica, permitindo que se retornasse a fase anterior para a correção de erros identificados. Escolheu-se como plataformas-alvo os principais sistemas operacionais para dispositivos móveis atuais, Android e iOS, e as linguagens de programação Java e Swift, respectivamente. Após a elaboração de uma versão (para cada um dos dois sistemas operacionais) sem erros aparentes, pôde-se avançar para avaliação em uma situação real.

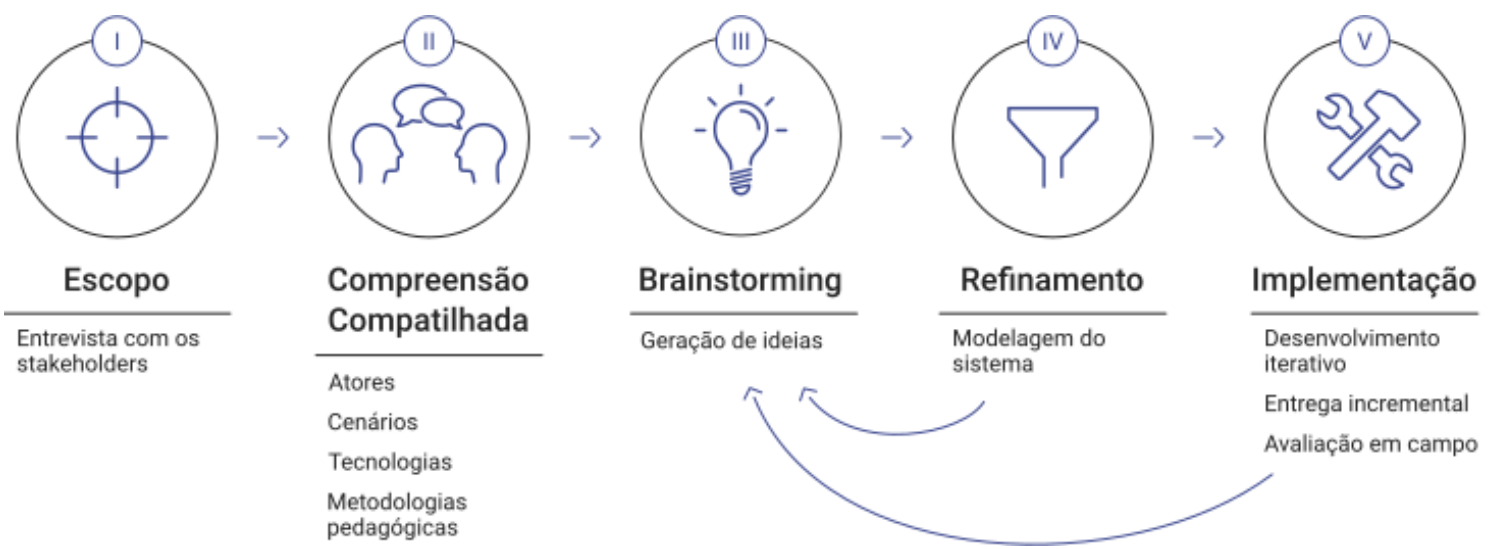

Figura 2 - Processo de desenvolvimento do Co-Design (Millard, 2009) adaptado.

\subsection{Utilização da Aplicação Móvel em Campo}

Em paralelo ao desenvolvimento da aplicação e antes dela ser testada pelos usuários finais, o projeto de pesquisa relacionado a ela foi cadastrado na Plataforma Brasil $^{1}$. Este sistema, criado pelo Governo Federal, consiste na base nacional unificada

\footnotetext{
${ }^{1}$ http://aplicacao.saude.gov.br/plataformabrasil/login.jsf
} 
de registro de pesquisas envolvendo seres humanos. Além disso, essa pesquisa também foi submetida e aprovada pelo comitê de ética do Centro Universitário Unichristus.

Para validar a aplicação, foi realizado um estudo de caso com 20 participantes distribuídos da seguinte forma: 15 estudantes do último ano de Medicina e 05 residentes em Anestesiologia. Os testes com a aplicação foram realizados durante uma semana no hospital Instituto Doutor José Frota (IJF), enquanto os participantes realizavam suas formações tanto no internato quanto na residência. O IJF é o maior centro de urgência e emergência de nível terciário da rede de saúde pública da Prefeitura de Fortaleza.

Para não ocupar muito tempo dos participantes e otimizar a realização dos testes, principalmente considerando o ambiente hospitalar onde se deu o estudo, foram levados smartphones e tablets com a aplicação já instalada para serem usados pelos aprendizes. A Figura 3.A) mostra um dos estudantes participantes utilizando a aplicação em um tablet com sistema operacional Android. A Figura 3.B) mostrar duas telas da aplicação no sistema operacional iOS, com informações sobre o estado físico do paciente e uma recomendação de conduta, respectivamente.
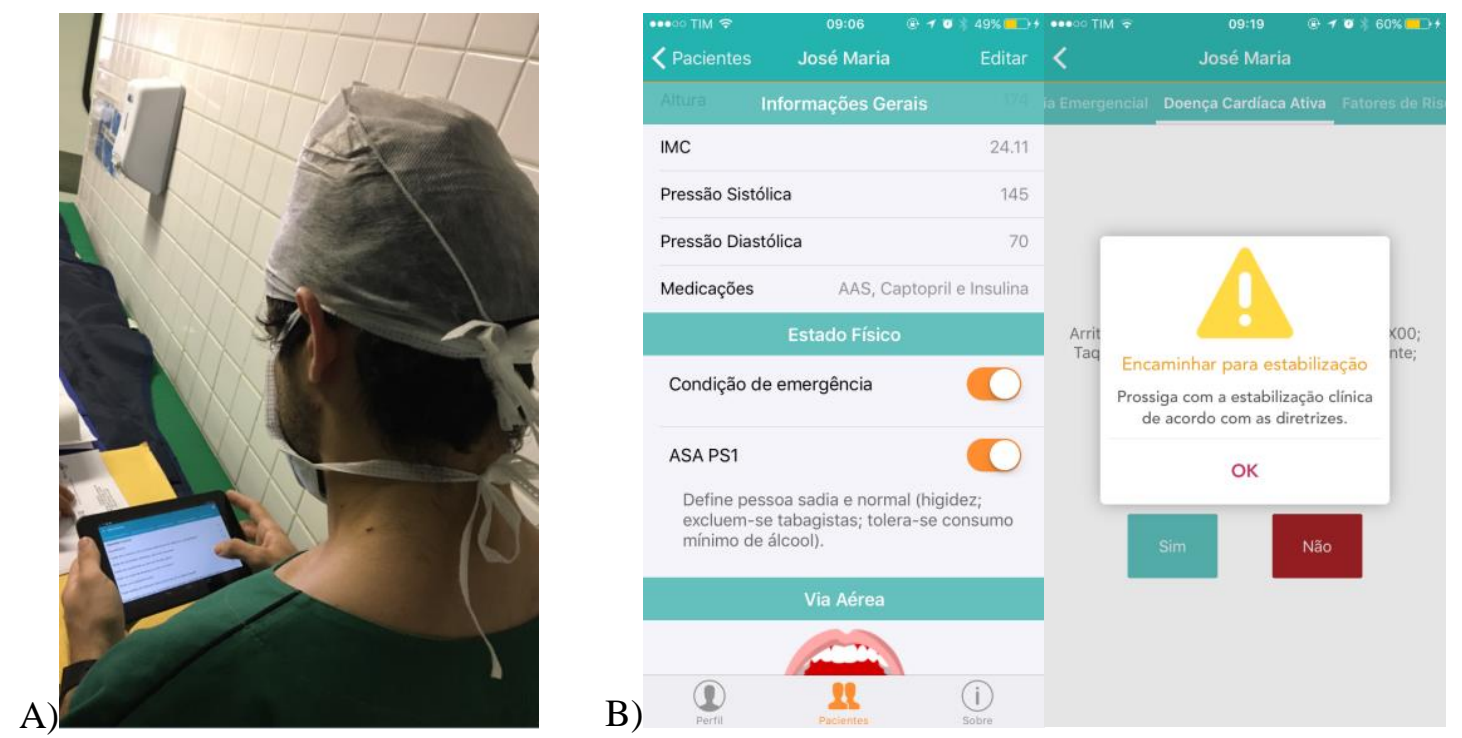

Figura 3 - A) Aluno de Medicina utilizando o aplicativo. B) Telas da aplicação móvel.

\subsection{Avaliação da Usabilidade e Utilidade}

Para avaliação da aplicação móvel foi desenvolvido um questionário padronizado que tinha como base outros questionários existentes, o qual foi dividido em quatro partes. A primeira, nomeada Parte 0 , serviu para coleta da autorização de utilização das respostas e de informações sobre o participante, como nível de escolaridade (se no internato ou na residência) e experiência no uso de aplicativos móveis.

Com a Parte 1, baseada no questionário SUS, pretendeu-se obter informações sobre a facilidade de uso (Usabilidade) e a simplicidade para se aprender a usar a aplicação (Capacidade de Aprendizado). A Parte 2, baseada no Modelo de Aceitação de Tecnologia de Davis (TAM), contou com 8 questões e era voltada para se identificar o nível de utilidade percebida pelos aprendizes sobre a aplicação (Utilidade Percebida).

As partes 0,1 e 2 eram compostas por questões objetivas, com opções para o participante selecionar. $\mathrm{Na}$ Parte 3, o participante tinha duas questões abertas onde poderia descrever suas opiniões quanto aos aspectos positivos, negativos e sugestões de melhorias para a aplicação. Foi solicitado a todos os participantes que não deixassem essas questões em branco, pois as respostas seriam utilizadas para a implementação de melhorias na aplicação. 


\section{RESULTADOS}

A Tabela 1 apresenta um resumo da análise sobre a Parte 1 do questionário de avaliação, que corresponde às questões baseadas na escala SUS. Os resultados demonstram que a aplicação recebeu uma boa avaliação de usabilidade, obtendo escore SUS médio igual a 90,6 (com desvio padrão de 9,2). Ao observar a classificação desse valor segundo dois estudos diferentes, o aplicativo móvel desenvolvido alcançou os níveis mais altos de usabilidade: nota A+, segundo Sauro e Lewis (2011) e nota A, na escala de Bangor, Kortum e Miller (2009).

Além disso, também se pode afirmar, com $95 \%$ de confiança, que o escore SUS para essa população está entre 86,3 e 94,9 (com margem de erro igual a 4,3). Para atestar a confiabilidade dos dados obtidos, utilizou-se o coeficiente alfa de Cronbach (Bonett; Wright, 2015). Como se pode observar na Tabela 1, valor obtido para esse coeficiente foi de 0,79 , caracterizando a amostra com tendo um bom nível de confiabilidade, dado que 0,70 é considerado o limite inferior para uma confiabilidade aceitável (Sauro, 2011).

Tabela1- Resumo da análise sobre a Usabilidade da aplicação $(\mathrm{N}=20)$.

\begin{tabular}{c|c|c|c}
\hline SCORE SUS & Desvio Padrão & $\begin{array}{c}\text { Intervalo de } \\
\text { Confiança }\end{array}$ & $\begin{array}{c}\text { Confiabilidade } \\
\text { da Amostra }\end{array}$ \\
\hline $\mathbf{9 0 , 6}$ & $\mathbf{9 , 2}$ & $\mathbf{8 6 , 3 - 9 4 , 9}$ & $\mathbf{0 , 7 9}$ \\
\hline
\end{tabular}

Diferentemente da escala SUS, o modelo de Davis não possui uma fórmula para obtenção de um valor único. Assim, a avaliação das respostas às questões da Parte 2 do questionário, relativas à utilidade percebida pelos aprendizes, se deu através da análise comparativa dos valores médios para cada questão e da frequência das respostas. $\mathrm{O}$ gráfico da Figura 4 mostra a frequência (\%) das respostas sobre a utilidade da aplicação para os alunos do internato e para os residentes em Anestesiologia.

Pode-se observar que os resultados demonstram que os aprendizes participantes do estudo concordam que a aplicação testada é útil para formação deles sobre o procedimento de avaliação pré-operatória. Destacam-se as questões 17 e 18, onde 100\% dos participantes concordam que a aplicação possibilita a sistematização da avaliação pré-operatória e que o conteúdo está em consonância com as melhores evidências

11) Facilitou-me a avaliação do risco preoperatório.

12) Dificultou-me a escrever textos e números.

13) Permitiu-me a identificar mais eficientemente as comorbidades dos pacientes.

14) Complicou a minha ident ificação das comorbidades dos pacientes.

15) É uma tecnologia útil para decisões quanto à necessidade de adiamento da cirurgia.

16) Ajudou-me na decisão quanto à necessidade de solicitação de exames adicionais.

17) Permitiu-me sistematizar a avaliação preoperatória em um intervalo de tempo satisfatório.

18) Contém informações atualizadas e em consonância com as me lhores evidências.

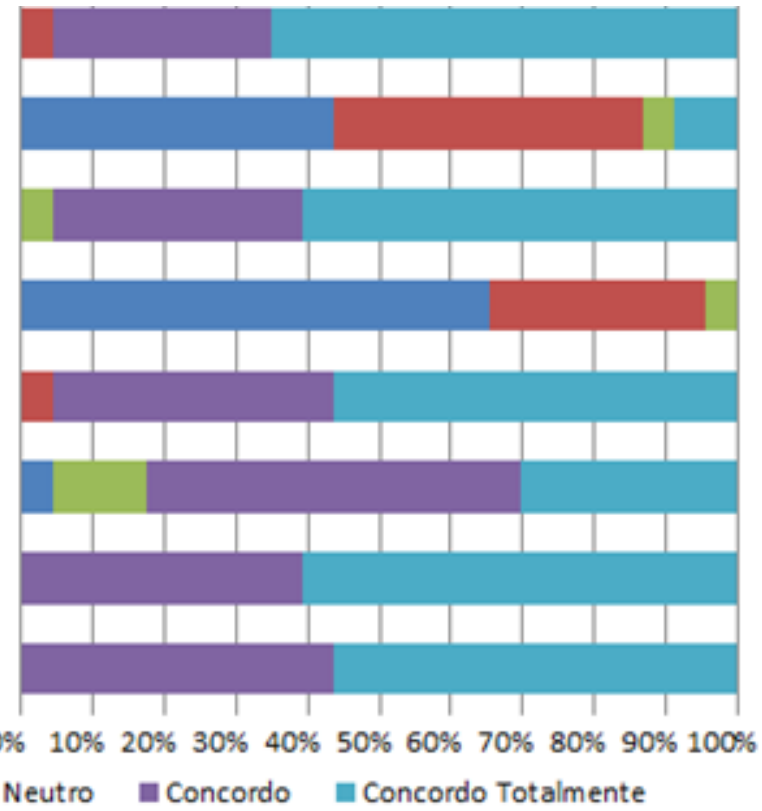

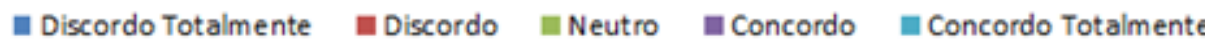

Figura 4 - Frequência da Utilidade Percebida pelos aprendizes sobre a aplicação. 
médicas atuais. As quais consistem em aspectos importantes na formação em Anestesiologia.

Além das questões objetivas, os participantes puderam escrever pontos positivos, negativos e sugestões para o aplicativo. As respostas confirmam a boa aceitação da aplicação móvel, destacando-se a importância para o treinamento e prática do procedimento de avaliação pré-operatória e para apoio à decisão sobre as condutas médicas. A seguir, são apresentadas algumas opiniões coletadas sobre o aplicativo:

"Facilita na identificação de riscos operatórios e ajuda na tomada de decisões através de um banco de dados de condutas."

"Sistematiza e otimiza a avaliação pré-operatória, poupando tempo através de perguntas objetivas.

"Torna a avaliação pré-operatória ágil, evita possíveis esquecimentos e facilita o acesso aos dados dos pacientes em momentos posteriores."

O aplicativo proporcionou aos alunos uma melhor compreensão, baseada em evidências da literatura médica atual, nos processos de tomada de decisão em situações de maior dificuldade. Por exemplo, em casos com pacientes com fatores de risco cardiovascular, a aplicação móvel direcionava para condutas específicas, tais como exames adicionais, adiamento ou suspensão de procedimentos, otimização de condições clínicas associadas ou encaminhamento para a cirurgia sem outras considerações.

\section{DISCUSSÃO}

O conteúdo necessário para o processo de tomada de decisões médicas é largamente auxiliado por diretrizes especializadas que, em geral, estão disponibilizadas em textos longos e que frequentemente tomam tempo excessivo. As soluções baseadas em dispositivos móveis oferecem aos estudantes e profissionais o acesso à informação no momento em que é requisitada de maneira que, independentemente do tempo e lugar, há disponibilização imediata. Além do mais, há atualmente uma preocupação com relação à baixa aderência a estas diretrizes que, por sua vez, pode causar omissão de terapias adequadas podendo contribuir para resultados indesejáveis no tratamento e maior dispêndio de recursos (Pronovost, 2013).

Segundo uma pesquisa realizada com 1210 participantes, entre estudantes e residentes de Medicina, o uso dos dispositivos móveis para seus estudos e pesquisas acadêmicas já está amplamente difundido (Dale Storie Mlis, 2014). Outros artigos apresentam estudos similares com aplicações móveis voltadas ao apoio à educação médica. Por exemplo, Cazella, Feyh e Ben (2014) demonstram a utilização de um aplicativo móvel, baseado em diretrizes clínicas, relacionado ao tratamento da tuberculose. O presente artigo se diferencia da maioria dos estudos relacionados por apresentar um trabalho que descreve desde o planejamento e desenvolvimento da aplicação móvel até a análise da sua usabilidade e utilidade para aprendizagem, no caso específico para o treinamento e prática do procedimento de avaliação pré-operatória.

Tendo em vista a multidisciplinaridade da equipe e o interesse em se obter uma aplicação final mais próxima do usuário final através da participação efetiva dos especialistas de domínio, optou-se pela utilização do modelo Co-Design de desenvolvimento de sistemas. Vale destacar que quatro fases, dentre as cinco do CoDesign, são voltadas para a identificação dos requisitos e definição dos modelos que determinarão como a aplicação deverá funcionar. Isso permitiu que o médico participasse mais ativamente da concepção do sistema, podendo colaborar em quatro 
fases do processo, e reduziu o impacto da sua falta de tempo, pois poderia contribuir em diferentes momentos.

Com relação à avaliação, buscou-se verificar se a aplicação móvel desenvolvida demonstrava facilidade no uso e qual era o grau de utilidade para aprendizagem e prática do procedimento de avaliação pré-operatória. Pôde-se observar que a aplicação foi aprovada nesses dois critérios, destacando-se o fato de promover um meio fácil, ubíquo e prático de suporte à decisão baseado em diretrizes clínicas. Dessa forma, a busca e utilização do conhecimento no momento e contexto apropriados potencializam o processo de aprendizagem e diminuem a possibilidade de omissões. Vale ressaltar que alguns dos usuários avaliados sugeriram acréscimos ao aplicativo tais como a inserção de registro gráfico de exames de eletrocardiograma, resultados do coagulograma e exames de imagem (radiografia).

\section{CONCLUSÃO}

Este artigo apresentou o processo de desenvolvimento, a utilização e a avaliação de uma aplicação móvel voltada para apoiar a formação na área de Anestesiologia. Foram descritos o uso da metodologia Co-Design para criação do aplicativo, os passos realizados para sua utilização em um ambiente hospitalar e os critérios e cálculos estatísticos utilizados para avaliação da usabilidade e utilidade percebida por alunos de Medicina e residentes em Anestesiologia.

A partir da análise realizada após a utilização em campo, verificou-se que a aplicação desenvolvida apresentou alto nível de usabilidade e foi considerada útil pelos participantes do estudo para o treinamento e a prática do procedimento de avaliação préoperatória. Os resultados também indicaram que aprimoramentos necessitam ser realizados para se obter uma ferramenta educacional mais abrangente. Portanto, como trabalho futuro, pretende-se realizar a implementação dessas novas funcionalidades e, posteriormente, disponibilizar a aplicação para o uso por parte de outros pesquisadores em outras instituições de ensino e hospitais. Com isso, também se planeja medir os ganhos proporcionados pela aplicação no processo de aprendizagem, que não foram previstos nesse estudo.

\section{REFERÊNCIAS}

BANGOR, Aaron; KORTUM, Philip; MILLER, James. Determining what individual SUS scores mean: Adding an adjective rating scale. Journal of usability studies, v. 4, n. 3, p. 114-123, 2009.

BONETT, Douglas G.; WRIGHT, Thomas A. Cronbach's alpha reliability: Interval estimation, hypothesis testing, and sample size planning. Journal of Organizational Behavior, v. 36, n. 1, p. 3-15, 2015.

BRANCH, Robert Maribe. Instructional design: The ADDIE approach. Springer Science \& Business Media, 2009.

BRIZ-PONCE, Laura et al. Effects of mobile learning in medical education: a counterfactual evaluation. Journal of medical systems, v. 40, n. 6, p. 1-6, 2016.

CAZELLA, Sílvio Cézar; FEYH, Rafael; BEN, Ângela Jornada. Ambient Intelligence - Software and Applications. Advances in Intelligent Systems and Computing, v. 291, n. January 2014, p. 217-224, 2014.

DALE STORIE MLIS, M. A. Mobile devices in medicine: A survey of how medical students, residents, and faculty use smartphones and other mobile devices to find information. Journal of the Medical Library Association,v. 102,n.1, p.22, 2014. 
DAVIES, Bethany S. et al. Mobile Medical Education (MoMEd)-how mobile information resources contribute to learning for undergraduate clinical students-a mixed methods study. BMC medical education, v. 12, n. 1, p. 1, 2012.

FULLER, Richard; JOYNES, Viktoria. Should mobile learning be compulsory for preparing students for learning in the workplace?. British Journal of Educational Technology, v. 46, n. 1, p. 153-158, 2015.

GILMAN, Ekaterina et al. Towards user support in ubiquitous learning systems. IEEE Transactions on Learning Technologies, v. 8, n. 1, p. 55-68, 2015.

HWANG, Gwo-Jen; WONG, Lung-Hsiang. Guest Editorial: Powering Up: Insights from Distinguished Mobile and Ubiquitous Learning Projects across the World. Educational Technology \& Society, v. 17, n. 2, p. 1-3, 2014.

MACK, Robert L.; NIELSEN, Jakob (Ed.). Usability inspection methods. New York, NY: Wiley \& Sons, 1994.

MARÇAL, Edgar; ANDRADE, Rossana; RIOS, Riverson. Aprendizagem utilizando dispositivos móveis com sistemas de realidade virtual. Revista Novas Tecnologias na Educação, v. 3, n. 1, 2005.

MARÇAL, Edgar; ANDRADE, Rossana; VIANA, Windson. Aulas de Campo Ubíquas. In: Simpósio Brasileiro de Informática na Educação - SBIE. 2015. p. 150.

MILLARD, David et al. Co-design and co-deployment methodologies for innovative m-learning systems. Multiplatform E-Learning Systems and Technologies: Mobile Devices for Ubiquitous ICT-Based Education: Mobile Devices for Ubiquitous ICTBased Education, v. 147, 2009.

O'CONNOR, Siobhan; ANDREWSB, Tom. Using Co-Design with Nursing Students to for Clinical Training Create Educational Apps. Nursing Informatics: EHealth for All: Every Level Collaboration-From Project to Realization, v. 225, p. 334, 2016.

PREUVENEERS, Davy; NOVAIS, Paulo. A survey of software engineering best practices for the development of smart applications in Ambient Intelligence. Journal of Ambient Intelligence and Smart Environments, v. 4, n. 3, p. 149-162, 2012.

PRONOVOST, Peter J. Enhancing physicians' use of clinical guidelines. JaMa, v. 310, n. 23, p. 2501-2502, 2013.

SAURO, Jeff. A practical guide to the system usability scale: Background, benchmarks \& best practices. Measuring Usability LLC, 2011.

SAURO, Jeff; LEWIS, James R. Quantifying the user experience: Practical statistics for user research. Elsevier, 2012.

SHUIB, Munir et al. Designing an Intelligent Mobile Learning Tool for Grammar Learning (i-MoL). iJIM, v. 9, n. 1, p. 41-46, 2015.

SILVA, Bruno MC et al. Mobile-health: A review of current state in 2015. Journal of biomedical informatics, v. 56, p. 265-272, 2015.

SPÍNOLA, Rodrigo Oliveira; TRAVASSOS, Guilherme Horta. Towards a framework to characterize ubiquitous software projects. Information and Software Technology, v. 54, n. 7, p. 759-785, 2012.

SUNG, Han-Yu; HWANG, Gwo-Jen; CHANG, Ya-Chi. Development of a mobile learning system based on a collaborative problem-posing strategy. Interactive Learning Environments, v. 24, n. 3, p. 456-471, 2016.

WALLACE, Linda G.; SHEETZ, Steven D. The adoption of software measures: A technology acceptance model (TAM) perspective. Information \& Management, v. 51, n. 2, p. 249-259, 2014.

WALSH, Kieran. Mobile Learning in Medical Education: Review. Ethiopian journal of health sciences, v. 25, n. 4, p. 363-366, 2015. 